\title{
Recent Advances in the Observation and Analysis of Stellar Magnetic Fields
}

\author{
Steven Saar \\ Harvard-Smithsonian Center for Astrophysics \\ 60 Garden Street MS 58 Cambridge, MA 02138 USA
}

\begin{abstract}
There has been considerable progress recently in the study of magnetic fields on late-type stars. Advances in the theory include investigation of systematic effects, new and improved methods of analysis for unpolarized and circularly polarized lines, new models of stellar dynamos and of broadband linear polarization, and the first studies of the thermodynamic nature of stellar magnetic regions and their vertical structure. Observationally, there have been new measurements, particularly of young and active stars (including the first detection of a field on a pre-main sequence object), a new monitoring campaign, the first indications of the relative temperatures in stellar plages, and the first measurements of fields in a single stellar active region. I discuss the results in the framework of stellar activity and surface structure.
\end{abstract}

\section{Introduction}

Although the ink is barely dry on the most recent (last summer's) review of magnetic field measurements on cool stars (Saar, 1990a), considerable further progress has been made over the past year. Advances have been made in exploring sources of systematic error, new and improved techniques, further measurements, and in extending field measurements to new classes of objects. Partly as a result, the beginnings of a coherent picture of properties of magnetic regions on cool stars may be starting to emerge.

In this review, I will concentrate on these recent results. Integrating them with other recent work, I will update the main discoveries in the field to date, investigate trends in measurements, and summarize the clues gathered so far concerning the structure and characteristics of fields on cool stars. I also refer the reader to recent reviews by Saar (1990a), Linsky (1989), and Gray (1988). 


\section{Investigation of sources of systematic error}

Nearly all successful methods of measuring the magnetic parameters of cool stars have followed Robinson (1980), and modeled the differential broadening of absorption lines in unpolarized light. The most simple analyses treat the effect of a magnetic field on an observed line profile, $F$, as the sum of two components: $F=f F_{\mathrm{m}}(B)+(1-f) F_{\mathrm{q}}(0)$. Here, $F_{\mathrm{m}}$ is the line profile in magnetic regions (with a mean field strength $B$ ), which are assumed to uniformly cover a fraction $f$ of the stellar surface; $F_{\mathrm{q}}$ is the quiet region profile $(B=0)$. Lines of various magnetic sensitivities (Landé $g_{\text {eff }}$ ) are compared to determine $f$ and $B$, with low $g_{\text {eff lines }}$ [where $F_{\mathrm{q}}(0) \approx F_{\mathrm{m}}(B)$ ] often used as an initial estimate of $F_{\mathrm{q}}$.

Of course, many simplifying assumptions must be made in the analyses due to the lack of data about the nature of the unresolved stellar surface. Two major sources of systematic error in stellar magnetic measurements are possible gradients in $B$ with height and the unknown thermodynamic differences between $F_{\mathrm{q}}$ and $F_{\mathrm{m}}$. Up to now, studies have generally assumed that $\nabla B=0$ and the atmospheres making up $F_{\mathrm{m}}$ and $F_{\mathrm{q}}$ are identical. Much of the disagreement surrounding the $f$ and $B$ values of $\epsilon$ Eri, however, can be explained by a combination of a non-zero $\nabla B$ and differences in the height of the formation of the various magnetic lines used (Grossmann-Doerth and Solanki, 1990; see also Saar, 1990a). Sun et al. (1986) and Saar (1988a) made some preliminary investigations of the effect of non-identical atmospheres. More recently, Basri et al. (1990) have studied these problems with more physically rigorous models of the Fe I $7748 \AA\left(g_{\mathrm{eff}}=1.1\right)$ and $8468 \AA\left(g_{\mathrm{eff}}=\right.$ $2.5)$ lines. They constructed theoretical profiles from combinations of the HolwegerMüller quiet solar atmosphere with various empirical plage and spot models. They find that fits to these, assuming identical atmospheres for $F_{\mathrm{m}}$ and $F_{\mathrm{q}}$, can lead to underestimates of $f B$ by as much as $40 \%$. Intriguingly, Basri et al. found they could not simultaneously fit $\lambda 7748$ and $\lambda 8468$ profiles computed with the plage + quiet solar atmosphere if the model assumed identical $F_{\mathrm{m}}$ and $F_{\mathrm{q}}$ atmospheres. I will discuss some implications of this in $\S 5$.

Most stellar measurements to date have used simpler atmospheric models than those of Basri et al. S. Solanki and I have recently been exploring the errors in the derived $f$ and $B$ values when realistic, self-consistent atmospheres embedded with fluxtubes are analyzed using a simple Unno (1956) type code and a MilneEddington atmosphere (e.g., Saar, 1988a; Bopp et al., 1989). We have calculated full radiative transfer along rays passing through a two-dimensional fluxtube + quiet atmosphere model (Steiner and Pizzo, 1989), and constructed theoretical profiles by summing over all rays. For our initial tests, we computed strong, simple triplet Fe I lines with $g_{\mathrm{eff}}=1$ and 2.5 at disk center with constant microturbulence. Since in practice, $\mathrm{Fe} / \mathrm{H}$, the oscillator strengths $(\log g f)$ and the collisional broadening must usually be adjusted, we adopted the following procedure. First, the low $g_{\text {eff }}$ line was fit (minimizing $\chi^{2}$ ) with the line opacity $\left(\eta_{0}\right)$, source function slope, and collisional broadening (Voigt $\alpha$ ) as free parameters. These values then became initial guesses in a fit of the high $g_{\text {eff }}$ line with $\eta_{0}, f$ and $B$ free. A final fit was then made to both lines simultaneously, with $\eta_{0}, \alpha, f$, and $B$ free. Sample 
Table 1. Results of Unno-type Fits to 2-D Atmosphere Line Models with $f \approx 0.30$

\begin{tabular}{|ll|ccccccc|}
\hline $\begin{array}{l}\text { quiet* } \\
\text { atm. }\end{array}$ & $\begin{array}{l}\text { mag.* } \\
\text { atm. }\end{array}$ & $\begin{array}{c}\lambda \\
(\AA)\end{array}$ & $\log g f$ & $\begin{array}{c}\chi_{L} \\
(\mathrm{eV})\end{array}$ & $\begin{array}{c}B(\tau=1) \\
(\mathrm{G})\end{array}$ & $\begin{array}{c}B(\tau=0.01) \\
(\mathrm{G})\end{array}$ & $\begin{array}{c}B \text { (fit) } \\
(\mathrm{G})\end{array}$ & $\begin{array}{c}f(\mathrm{fit}) \\
(\%)\end{array}$ \\
\hline HSRA & NET & 6000 & -5.58 & 0 & 2000 & 970 & 1240 & 13 \\
HSRA & NET & 6000 & -3.54 & 2 & 2000 & 970 & 1360 & 15 \\
HSRA & NET & 6000 & -1.60 & 4 & 2000 & 970 & 1450 & 18 \\
HSRA & NET & 16000 & 0.07 & 5 & 2000 & 970 & 1380 & 17 \\
HSRA & NET & 22000 & -0.70 & 5 & 2000 & 970 & 1170 & 15 \\
\hline HSRA & HSRA & 6000 & -5.58 & 0 & 2000 & 1030 & 1460 & 24 \\
HSRA & HSRA & 6000 & -3.54 & 2 & 2000 & 1030 & 1510 & 25 \\
HSRA & HSRA & 6000 & -1.60 & 4 & 2000 & 1030 & 1530 & 25 \\
HSRA & HSRA & 16000 & 0.07 & 5 & 2000 & 1030 & 1520 & 30 \\
HSRA & HSRA & 22000 & -0.70 & 5 & 2000 & 1030 & 1350 & 31 \\
\hline K2V & K2V & 6000 & -5.58 & 0 & 3195 & 1750 & 2100 & 27 \\
K2V & K2V & 6000 & -3.54 & 2 & 3195 & 1750 & 2070 & 33 \\
K2V & K2V & 6000 & -1.60 & 4 & 3195 & 1750 & 2210 & 31 \\
K2V & K2V & 16000 & 0.07 & 5 & 3195 & 1750 & 2500 & 28 \\
K2V & K2V & 22000 & -0.70 & 5 & 3195 & 1750 & 2240 & 31 \\
\hline
\end{tabular}

References: HSRA = Harvard-Smithsonian Ref. Atm.; NET = network model

(Solanki 1986); K2V = K2 dwarf model (Basri and Marcy 1988)

results are given in Table 1 , for the case of $f \approx 0.30$ at an "average" level of line formation.

A detailed interpretation of the results is not possible at present, as we have not yet calculated the "correct" $B$ values where the lines are formed. Still, some general comments can be made. To first order, the derived $B$ values appear to be reasonable $\left[B\left(\tau_{5000}=0.01\right)<B\right.$ (fit $\left.)<B\left(\tau_{5000}=1\right)\right]$. Qualitatively, we obtain underestimates of $f$ and $f B$ similar to Basri et al. in the case of the solar + network model. For the same model, the magnetic flux density $(=f B)$ inferred from $\lambda 6000$ increases as a function of excitation potential $\left(\chi_{L}\right)$, consistent with a stronger contribution to the line from the (hotter) magnetic portion of the atmosphere for lines of higher $\chi_{L}$ (see Grossmann-Doerth and Solanki, 1990). Fits were poorer for the infrared models, where the effects of $\nabla B$ on the Zeeman sigma components were evident. We plan to extend this work in the near future, studying center-tolimb effects and disk-integrated models with various analysis methods.

\section{Improved analysis methods and new measurements}

As the above analyses suggest, one way to investigate the differences between $F_{\mathrm{m}}$ and $F_{\mathrm{q}}$ (which are not even well determined in the Sun; e.g., Stenflo, 1989) is to analyze lines with various $\chi_{\mathrm{L}}$ and $\log g f$, thus probing different heights of formation and sensitivities to density and temperature. An approximate analysis along these lines has been completed by Mathys and Solanki (1989). They applied the Stenfo and Lindegren (1977) method (which determines $f$ and $B$ from a regression analysis correlating line widths and strengths with $\chi_{\mathrm{L}}$ and a modified $g_{\text {eff }}$ ) to several cool stars. For the well-studied K2 dwarf, $\epsilon$ Eri, they find evidence that the magnetic regions are somewhat warmer than the quiet photosphere. While this result represents the first direct evidence of the thermodynamic differences between $F_{\mathrm{m}}$ and $F_{\mathrm{q}}$, one would ultimately like to test the results against magnetic measurements made using more rigorous analyses (spectrum synthesis). 
Valenti (1991) has recently begun such a study, using the Hamilton Echelle at Lick to obtain over $2000 \AA$ of spectrum at moderate resolution $(\approx 40,000)$. Simultaneously fitting $25 \mathrm{Fe} I$ lines in the active G8 dwarf $\xi$ Boo A, he finds $B \approx 2000 \mathrm{G}$ and $f \approx 0.20$, generally consistent with previous results (Marcy and Basri, 1989; Saar, 1990a). At the moment, Valenti (1991) is using identical, scaled Holweger-Müller atmospheres for both $F_{\mathrm{q}}$ and $F_{\mathrm{m}}$, and a coarse grid of parameters, but eventually analysis of the fit residuals as a function of $\chi_{\mathrm{L}}$ and $\log g f$ should indicate how $F_{\mathbf{q}}$ and $F_{\mathrm{m}}$ differ.

Another method of studying the quiet/active region differences has been proposed by Sánchez Almeida and García López (1991). They focus on two lines (Fe I $5247 \AA$ and $5250 \AA$ ) which are nearly identical (e.g., $\Delta \log g f \approx 0.008$ ) except for $g_{\text {eff }}$. Subtraction of one profile from the other should thus very nearly cancel $F_{\mathrm{q}}$, leaving only a residual $\Delta F_{\mathrm{m}}$. The authors model $\Delta F_{\mathrm{m}}$ using a Holweger-Müller atmosphere, including disk-integration, known blends, and magneto-optical (MO) effects (which may be important in some cases; Landolfi et al. 1989). Preliminary results for stars were somewhat confusing, however, with similar magnetic fluxes found for both $\lambda$ Ser (G0V, inactive) and $\xi$ Boo A (G8V, active). The problem may lie in the model atmosphere ( $c f$. Valenti, 1991) or the disk-integration: analysis of solar plage yields reäsonable results, but fits to $\Delta F_{\mathrm{m}}$ in solar flux spectra show a spuriously large $f B$ values (Sánchez Almeida, private comm.).

Unidentified blends may also be a source of confusion, stressing the need for accurate $\log g f$ values for all lines and blends in the regions to be modeled. Unfortunately, the $g f$ data are often incomplete or of insufficient accuracy. For stars with $T_{\text {eff }}$ similar to the Sun, one can circumvent the problem by deriving empirical $g f$ values from solar spectra (e.g., Valenti, 1991). This strategy fails for stars significantly cooler than the Sun, however, where many lines which are weak or absent in the Sun become important. Saar et al. (1990) have developed a method to obtain approximate $g f$ values for lines in cooler active stars by carefully selecting comparison stars of similar $T_{\text {eff }}$ but very low magnetic activity levels (see also Saar, 1990b). In our test study, the low activity comparison HD $32147\left(\mathrm{P}_{\text {rot }} \approx 47\right.$ days) proved a nearly identical match to the K5Ve BY Dra star BD $+26^{\circ} 730$ once broadened to the same $v \sin i$. The only exception in over $20 \AA$ of spectrum was at the lone high $g_{\text {eff }}$ line (Fe I $6173 \AA ; g_{\text {eff }}=2.5$ ), indicating the presence of substantial magnetic flux. The $\log g f$ values found for HD 32147 were applied to a magnetic field analysis of $\mathrm{BD}+26^{\circ} 730$ (using the disk-integrated model of Bopp et al. 1989), yielding $B=2600 \mathrm{G}$ and $f=0.50$. I recently recalculated the $\mathrm{BD}$ $+26^{\circ} 730$ spectrum including MO effects, using Calamai et al. (1975). Changes in the $6173 \AA$ line amounted to $\leq 0.05 \%$, implying the derived $f$ and $B$ are negligibly affected by $\mathrm{MO}$ effects in this case. A similar, preliminary analysis of the young K2 dwarf HD 17925 yielded $B \approx 1500 \mathrm{G}$ and $f \approx 0.35$ (Saar, 1990b).

All of the preceding analyses have difficulties measuring $f$ and $B$ on stars where $v \sin i \geq 10 \mathrm{~km} \mathrm{~s}^{-1}$, due to problems discerning the small magnetic broadening component in lines strongly smeared by rotation. But while it may be difficult to actually separate $f$ and $B$, it is still possible to see some effects of the magnetic fields. The Zeeman effect redistributes opacity away from line center, so in opti- 
cally thick lines, magnetic fields will increase the equivalent width. This so-called "magnetic intensification" effect (e.g., Leroy, 1962) is being employed by Basri and Marcy (1991) to search for evidence of magnetic flux on weak-lined T Tauri stars. The basic strategy is to look for deviations from expected equivalent width (for $B=0)$. They find $f B \approx 1000 \mathrm{G}$ on Tap 35 , a G8 T Tauri $\left(v \sin i \approx 17 \mathrm{~km} \mathrm{~s}^{-1}\right)$, the first field measurement for this important class of stars.

White light spectra (Stokes $I$ ) have almost exclusively been used for determinations of $f$ and $B$ on cool stars, since the mixed polarities present on stars effectively cancel the integrated circular polarization (Stokes $V$ ) in most cases (e.g., Borra et al., 1984). Similarly, complex magnetic topologies reduce linear polarization (Stokes $Q$ and $U$ ) to small levels $\left(\leq 10^{-3}\right)$ while complicating its interpretation (see Huovelin and Saar, 1991). Recently, however, Semel (1989) has proposed a new kind of analysis employing Stokes $V$ profiles which can extract magnetic field information from rapidly rotating stars. The technique, dubbed "Zeeman Doppler imaging", relies on the stars' rapid rotation to separate magnetic regions of opposite polarity in velocity, thereby preserving a measurable residual in the integrated signal. The method thus measures the net magnetic flux in a given velocity resolution element. Donati et al. (1990) have employed the technique to measure a net unipolar filling factor of $14 \%$ at one rotational phase of the primary (K1 IVe) star in the RS CVn system HR 1099 , assuming $B \equiv 1000 \mathrm{G}$. This implies $f \geq 0.28$ for a bipolar geometry ( $I$ use this value in $\S 4$ ). The shape of the $I$ profile at the position of the $V$ profile signature indicates that the region was bright relative to the quiet photosphere.

\section{Trends in the measurements}

I now take the new magnetic measurements and combine them with previous data (from Saar, 1990a) to update the trends seen between $f$ and $B$ and various stellar parameters. The new data are compiled in Table 2; due to the aforementioned calibration problems, the results of the line subtraction method have been omitted. I have estimated the rotational period, $\mathrm{P}_{\text {rot }}$, of Tap 35 from its $v \sin i$ and radius (Walter et al., 1988). Convective turnover times, $\tau_{\mathfrak{c}}$, were taken from Stẹpień (1989) for dwarfs, Basri (1987) for RS CVns, and Gilliland (1985) for Tap 35. For latter two groups, $\tau_{\mathrm{c}}$ was normalized to Stępien's $\tau_{\mathrm{c}}(\odot)=13.7$ days (note, this correction was not applied in Saar, 1990a). Random errors in the $f$ and $B$ measurements are generally $\leq 0.1$ dex; poorly known systematic effects (see $\$ 2$ ) dominate the uncertainties. A good part of the spread in the relationships discussed below are likely due these, plus the use of differing techniques and lines. The absolute scale of $f$, in particular, is uncertain. Finally, $f B$ and $\sqrt{f} B$ are better determined than either $f$ or $B$ separately (Gray, 1984; Saar, 1988a; Basri et al., 1990).

With these caveats in mind, I first consider relationships between $B$ and stellar parameters. Saar and Linsky (1986) first noted that $B$ seemed to correlate well with the gas pressure equipartition field $B_{\text {eq }} \propto P_{\text {gas }}^{0.5}$. Values of $B_{\text {eq }}$ were computed using the formulation of Saar (1990a): $B_{\text {eq }}=B_{\odot} \times\left(P_{\text {gas }, *} / P_{\text {gas }, \odot}\right)^{0.5} \mathrm{kG}$, where $B_{\odot} \equiv$ 
Table 2. Summary of Recent Magnetic Field Measurements

\begin{tabular}{|c|c|c|c|c|c|c|c|c|c|}
\hline Star & Sp. Type & $\overline{B-V}$ & $\begin{array}{c}B \\
(\mathrm{kG})\end{array}$ & $\begin{array}{c}f \\
(\%)\end{array}$ & Ref. & $\begin{array}{l}\text { adopted } \\
B_{\text {eq }}(\mathrm{kG}) \\
\end{array}$ & $\begin{array}{l}\text { Prot } \\
\text { (days) }\end{array}$ & $\begin{array}{c}\tau_{c} \\
\text { (days) }\end{array}$ & $\begin{array}{c}\text { age } \\
\text { (Gyr) }\end{array}$ \\
\hline HD 10700 & G8V & 0.72 & $\ldots$ & $\ldots$ & 1 & 1.7 & 31.9 & 16.9 & $\ldots$ \\
\hline HD $131156 \mathrm{~A}$ & G8V & 0.76 & 2.0 & 20 & 1 & 1.7 & 6.2 & 19.0 & $0.3^{a}$ \\
\hline Tap 35 & G8 & 0.79 & $f B \approx$ & $1.0 \mathrm{kG}$ & 2 & 1.4 & $\approx 3.5$ & $\approx 40$ & $0.008^{b}$ \\
\hline HD 17925 & $\mathrm{~K} 2 \mathrm{~V}$ & 0.87 & 1.5 & 35 & 3 & 2.2 & 6.6 & 20.5 & $0.07^{c}$ \\
\hline $\mathrm{BD}+26^{\circ} 730$ & $\mathrm{~K} 5 \mathrm{Ve}+$ & 1.12 & 2.6 & 50 & 4 & 2.6 & 1.85 & 20.5 & $0.7^{d}$ \\
\hline HR 1099 & K1IVe+ & 0.92 & $\equiv 1.0$ & $\geq 14$ & 5 & 1.1 & 2.84 & $\approx 50$ & $\ldots$ \\
\hline
\end{tabular}

$1500 \mathrm{G}$ and $P_{\text {gas }}$ is taken at some reference level in the photosphere (e.g., $\tau_{5000}=$ 1). I have taken $f_{\odot}=0.02$. For Tap 35 and HR 1099, I used the main sequence $B_{\text {eq }}$ for the appropriate spectral type and scaled this by a factor $\left(g_{*} / g_{M S}\right)^{0.3}$ (where $\mathrm{g}$ is the surface gravity), since $P_{\mathrm{gas}} \propto \mathrm{g}^{0.6}$ (Gray, 1976). Figure 1 shows that in general, $B \leq B_{\text {eq }}$ (with the possible exception of some of the RS CVn data), consistent with Saar (1990a) and theoretical estimates (Galloway and Weiss, 1981; Zwaan and Cram, 1989). There is no correlation between $B$ and $f$ or $\sqrt{f}$, as would be expected if errors in separating $f$ and $B$ dominated the results.

Dynamo theories and stellar activity diagnostics strongly imply that some combination of magnetic field-related parameter(s) should be a function of the stellar rotation rate. The field strength, however, does not correlate strongly with either normalized angular velocity, $\Omega\left(\equiv \mathrm{P}_{\text {rot }}^{-1}\right.$; Fig. 1$)$ or inverse Rossby number, $\tau_{\mathrm{c}} \Omega$. (The apparent increase in $B$ for $\log \Omega>-0.7$ and $\log \tau_{\mathrm{c}} \Omega>0.5$ is likely a selection effect caused by the lack of $\mathrm{M}$ dwarfs detected for small $\Omega$ and the inability to measure small $B$ values when $v \sin i$ is large).
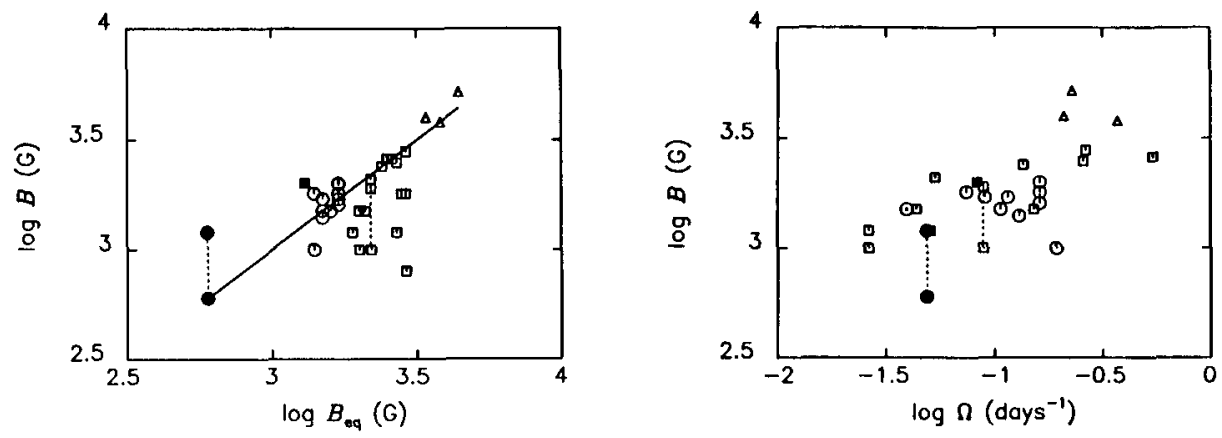

Fig. 1. $B$ vs. $B_{\text {eq }} \propto P_{\text {gas }}{ }^{0.5}$ (left) and $B$ vs. $\Omega$ (right). Circles, squares and triangles represent $G, K$, and $M$ stars; open symbols are dwarfs and filled are lower gravity stars; the Sun is denoted by $\odot$. Different measurements of the same object are connected by dotted lines and the solid line depicts $B=B_{\text {eq }}$. See text for details.

The magnetic flux density, $f B$, on the other hand, shows significant correlations with $\Omega$ both and $\tau_{\mathrm{c}} \Omega$ (Figs. 2 and 3). Least-squares power law fits $\left[f B \propto \Omega^{\alpha}\right.$ or $\left.\left(\tau_{\mathrm{c}} \Omega\right)^{\alpha}\right]$ yield essentially linear relationships, although modified fits $(e . g$. , giv- 
ing the Sun greater weight, or ignoring some $\mathrm{K}$ stars with more uncertain $f B$ values; see Marcy and Basri, 1989) can increase $\alpha$ to 1.5 or 2.0. The correlation with $\tau_{\mathrm{c}} \Omega$ yields somewhat smaller residuals. Three of the new measurements (BD $+26^{\circ} 730$, HR 1099 and Tap 35) plus AD Leo combine to give indicate a possible "saturated" $f B$ level for $\log \tau_{\mathrm{c}} \Omega>0.75$ (note that $f B_{\mathrm{HR} 1099} \leq 1.0 \mathrm{kG}$ if its $B \leq$ $B_{\text {eq }}$; Donati et al., 1990). Further data for rapid rotators are needed to confirm the existence of this saturation level, which has been predicted by a recent dynamo model (MacGregor and Brenner, 1989). Montesinos et al. (1991) have had some success matching measured $f B$ values for $\mathrm{G}$ and $\mathrm{K}$ dwarfs by using an improved Durney and Robinson (1982) dynamo model.

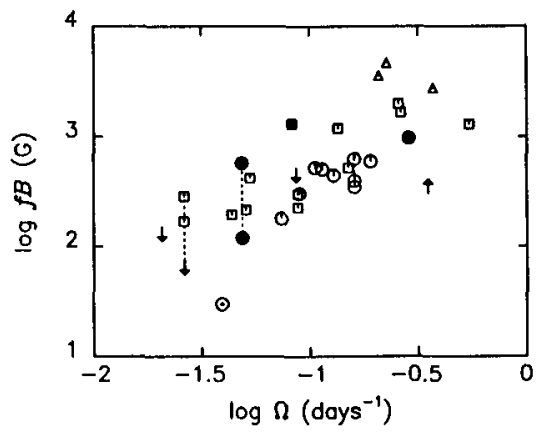

Fig. 2. $f B$ vs. $\Omega$ (symbols as in Fig. 1 ; see text for details).

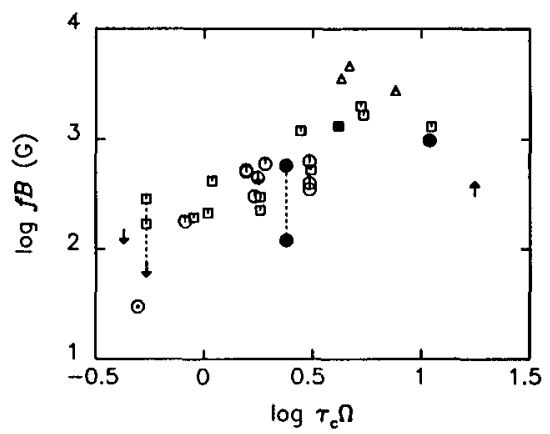

Fig. 3. $f B$ vs. $\tau_{\mathrm{c}} \Omega$ (symbols as in Fig. 1 ; see text for details).

Since $B$ is apparently not a function of rotation, the correlations between rotation and $f B$ are thus the result of a connection between rotation and $f$. This inference is borne out by the magnetic data (Fig. 4; where I have assumed $B_{\text {Tap35 }}$ $=B_{\text {eq }}$, so that $f_{\text {Tap35 }} \approx 0.70$ ). The relationship between $f$ and $\tau_{\mathrm{c}} \Omega$ is significantly tighter than for $f$ and $\Omega$. A saturated level is clearly seen for $\log \tau_{c} \Omega>0.5$ to 0.75 , roughly consistent with the $\tau_{\mathrm{c}} \Omega$ at which magnetic activity "saturates" (e.g., Vilhu, 1984). For $\log \tau_{\mathrm{c}} \Omega<0.75, f \propto\left(\tau_{\mathrm{c}} \Omega\right)^{\alpha}$ with $0.8 \leq \alpha \leq 1.8$ (depending on e.g., how strongly the Sun is weighted). An important implication of these results is that rotation governs magnetic activity by affecting the area coverage of active regions and not their field strengths.

Two of the new detections (Tap 35 and HD 17925) significantly extend the range of stellar ages $(t)$ over which $f$ and $B$ have been measured. The well known decline in magnetic activity with time (e.g., Hartmann and Noyes, 1987) is clearly reflected in $f B$ (Fig. 5, updating Fig. 2 from Saar, 1990b). An exponential decay law provides the best fit: $f B(G)=840 e^{-0.82 t(G y r)}$. The decline of activity with age is thus a direct result of a decline in $f$ with stellar age. The spread of activity at a given age may then partly be due to the range of $B$ at a fixed $t$.

It is natural to also look for relationships between $f B$ and the activity diagnostics (e.g., chromospheric and coronal emission) which long have been used as surrogates for the magnetic flux itself. Figure 6 (from Saar, 1990b) shows the result of an analysis of the magnetic, $\mathrm{X}$-ray $\left(F_{X}\right)$, and residual $\mathrm{Ca}$ II $\left(\Delta F_{C a I I}\right)$ 


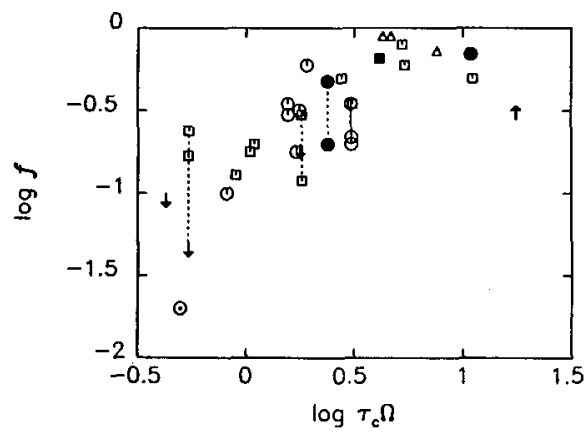

Fig. 4. $f$ vs. $\tau_{\mathrm{c}} \Omega$ (symbols as in Figure 1 ; see text for details).

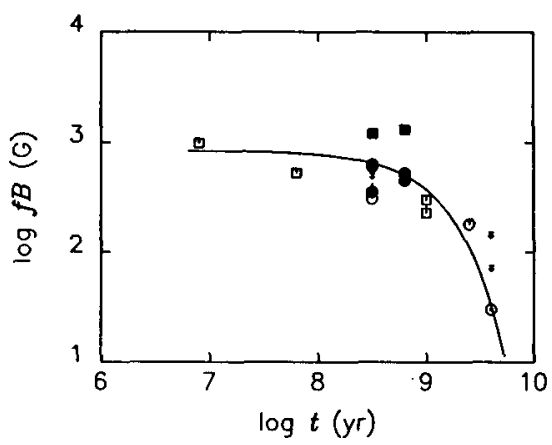

Fig. 5. $f B$ vs. age $t$. Here, filled symbols represent cluster members, open symbols have ages based on $\mathrm{Li} \mathrm{I}$. An exponential fit is shown (solid; see text).

flux densities. Clear correlations are seen, which, combined with studies of the transition region (TR) C IV line (Saar, 1988b; Schrijver, 1990a) appear to define a sequence (chromosphere-TR-corona) of power laws (Schrijver, 1990b): $\Delta F_{C a I I} \propto(f B)^{0.5}, F_{C I V} \propto(f B)^{0.7}$, and $F_{X} \propto(f B)^{1.0}$. (Including the new data for Tap 35 and HR 1099 has little effect on the derived power laws). These relations are consistent with flux-flux relations for stars and for $f B$-flux relations in spatially averaged solar regions (e.g., Schrijver et al. 1989). Some implications for atmospheric heating are discussed in Saar (1990b) and Schrijver (1990a,b).
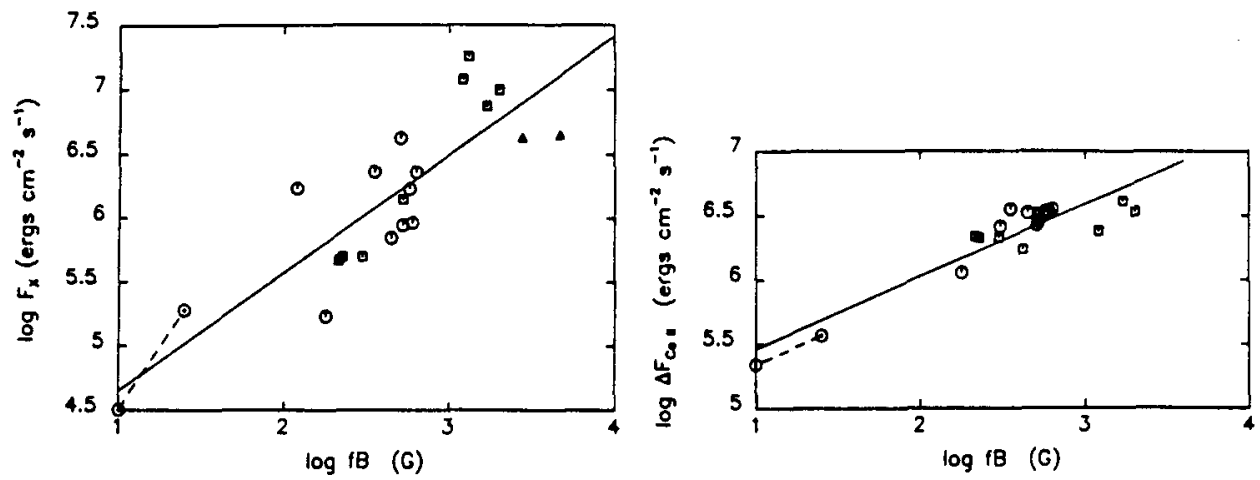

Fig. 6. $f B$ vs. $F_{X}$ (left) and $\Delta F_{C a I I}$ (right). Symbols as in Fig. 1, except dwarfs and low gravity stars are not distinguished. The best fit power laws, $\Delta F_{C a I I} \propto(f B)^{0.56}$ and $F_{X} \propto(f B)^{0.92}$, are shown (solid). From Saar (1990b). 


\section{Clues to the nature of stellar magnetic regions}

The $\mathrm{BD}+26^{\circ} 730$ field measurement $(\S 3)$ was made during an extensive observing campaign which studied the star over several rotations in 1988 (Saar et al., 1990). No variability (outside of flares) in activity or $f B$ was detected over three weeks. This was not unexpected; the star's low inclination $\left(i \leq 20^{\circ}\right)$ implies that any changes in activity would almost certainly be due to evolution of active regions (AR) rather than rotational modulation. However, we also found no change in the level of $\mathrm{H} \alpha$ or UV line emission between our data and observations in early 1981 - despite the fact that $\mathrm{BD}+26^{\circ} 730$ became significantly fainter (more spotted) during this period as it approached starspot cycle minimum (Hartmann et al., 1981). While a lack of correlation between activity and spot coverage has been noted before in some cases (e.g. Butler et al., 1987), $\mathrm{BD}+26^{\circ} 730$ is the first case where $f$, and $B$ and an absolute starspot filling factor are also available $\left(f_{\text {spot }} \approx 0.20 ;\right.$ Saar and Neff, 1990).

Since starspots contribute little to Zeeman line profiles in the optical (e.g., Saar, 1988a), the fields detected on cool stars must reside in "brighter" AR - the stellar analog of plage and network. This "brighter" component probably always dominates the total $f$ in stars. $\mathrm{BD}+26^{\circ} 730$ is thus nearly covered with $\mathrm{AR}$, with $f \approx f_{\text {plage }}=0.50$ and $f_{\text {spot }} \approx 0.20$. Perhaps because of this "saturated" level of $\mathrm{AR}$ (note the star's position in Fig. 4), BD $+26^{\circ} 730$ can increase $f_{\text {spot }}$ during its magnetic cycle only at the expense of $f_{\text {plage }}$, so that the total $f$ and associated activity levels remain constant. The creation of more magnetic flux then only leads to the coalescence of more plage into spots (having higher $B$; Mullan, 1984), with no net change in observed activity. Note that with "bright" $f_{\text {plage }}$ converting into $f_{\text {spot }}$ and vice versa, spot cycle photometric variations are increased over the normal assumption of quiet $\Leftrightarrow$ spot, helping to explain the large magnitude changes for some stars. Also, since $i \approx 20^{\circ}$, the large AR coverage must be near the poles, confirming the existence of high latitude AR on active stars ( $c f$. Vogt, 1988; Hackman et al., 1991). AR on stars with large $f$ also appear to be more highly organized than on the Sun - significant unipolar concentrations of flux suggest large coherent regions (Kemp et al., 1987; Donati et al., 1990).

But what does it mean to have $f \approx 50 \%$ or more? Clearly, the stellar surface must be considerably different from the Sun if we are to take $f$ at face value. Solanki and Steiner (1990) suggest that the solar chromospheric magnetic canopy may be formed closer to (or in) the photosphere in active cool stars, partly explaining the large $f$ values. The largest $f$ values are indeed seen among $\mathrm{K}$ and $\mathrm{M}$ dwarfs, where the lowering of the canopy is predicted to be largest. These same stars are often flare stars as well, consistent with the greater probability of energetic magnetic reconnection when $f$ is large (Saar et al., 1987).

Alternately, atmospheric differences between $F_{\mathrm{q}}$ and $F_{\mathrm{m}}$ not included in the in measurements may be causing overestimates of $f$. Stellar plage is apparently warmer than the surrounding photosphere (Mathys and Solanki, 1989; Simon et al., 1985; Radick et al., 1988) but it is unclear just how much warmer, or how this effect impacts $f$ determinations. Detailed comparison of quiet and active star spectra by 
Basri et al. (1989) indicate the situation is complex. Many lines show little change between stars of very different activity levels, suggesting the differences between $F_{\mathrm{q}}$ and $F_{\mathrm{m}}$ are not large, yet some strong lines show large differences. Recall, too, that Basri et al. (1990) were unable to fit theoretical profiles, computed with a (hot) Solanki (1986) network model for $F_{\mathrm{m}}$, with models having identical $F_{\mathrm{q}}$ and $F_{\mathrm{m}}$ atmospheres (see §2). The typical ease of fitting stellar data with the same $F_{\mathrm{q}}=$ $F_{\mathrm{m}}$ models (Marcy and Basri, 1989) then suggests that AR on stars differ less from $F_{\mathrm{q}}$ than their solar counterparts. Consistent with this idea, the smaller temperature differences seen in solar plage relative to network (e.g., Keller et al. 1990) imply that as $f$ increases, relative $F_{\mathbf{q}}-F_{\mathbf{m}}$ differences decrease. These ideas suggest that current $f$ measurements may not need significant revision.

Another possibility, however, is that since the simple two component picture of stellar surfaces used is incomplete, proper revision of the models would reduce $f$ (see also Marcy et al., 1991). An important part of fluxtube models is a dark, nonmagnetic downflowing region immediately outside (e.g., Grossmann-Doerth et al., 1988). Inclusion of such a component in stellar models may be useful in a number of ways. First, the dark circumtubular (CT) area could balance a hot $F_{\mathrm{m}}$, leading to little net change in most lines or overall spectral type-color relations (Basri et al., 1989; 1990). The hot $F_{\mathrm{m}}$ atmosphere might then imply a significant reduction in measured $f$ values (due to underestimated continuum and overestimated line strengths in the $F_{\mathrm{q}}=F_{\mathrm{m}}$ analyses).

Cool stellar CT regions may also have an observational counterparts ( $c f$. Brandt and Solanki 1990). While their restricted $g_{\text {eff }}$ range and simplified magnetic analysis may have prevented detection of $f B$ in surrounding AR (Saar, 1990a; Saar et al., 1988), the "starpatch" model of Toner and Gray (1988) for the active dwarf $\xi$ Boo A shows strong similarities to CT downflows (cool temperatures, enhanced velocities and closely associated Ca II emission; see also Toner and LaBonte, 1990). Convection is suppressed in magnetic regions on the Sun (e.g., Livingston, 1982). It seems possible that on very active stars, convection is restricted over such a large area that it must be enhanced in the remaining quiet/CT regions. Such enhancements could explain greater macroturbulence values (Gray, 1984) and the larger line bisector spans and variability (Bruning and Saar, 1990; Saar and Bruning, 1990) seen in active stars. Some fraction of the "spot" coverage on stars may in fact be due to the CT medium as well (thanks to G. Marcy for suggesting this).

Finally, it is not clear at the moment how low gravity stars (especially the RS CVns) fit in to the general scheme laid out in $\S 4$ (see Donati et al., 1990, and these Proceedings). They may have $B>B_{\text {eq }}$ and/or lower $f B$ than dwarfs at the same activity levels. Schrijver (1990b) has noted "over-active" stars among this class and suggests the need for additional parameters to fully describe their activity.

Many issues raised in this section remain unresolved and more questions beg to be answered. Clearly, further observations and model explorations are needed to extract more realistic $f$ and $B$ measurements and ultimately unravel the complex role of magnetic fields in the physics of cool stars. Significant progress has been made, however, and I think we may look forward to 1991 with hope for still more exciting revelations. 
This work has been supported by a Smithsonian Institution fellowship, NASA grant NAGW-221, and NSF grant INT-8900202. I grateful to NOAO and ESO for their generous allocation of telescope time. I would also like to offer special thanks to G. Basri, J.-F. Donati, G. Marcy, B. Montesinos, J. Sánchez Almeida, C. Schrijver, S. Solanki, C. Toner, and J. Valenti, for very helpful discussions and for kindly sharing results with me prior to publication.

\section{References}

Basri, G.S.: 1987, Astrophys. J. 316, 377

Basri, G.S., Marcy, G. W.: 1991, these Proceedings

Basri, G.S., Marcy, G. W., Valenti, J.: 1990, Astrophys. J. 360, 650

Basri, G.S., Wilcots, E., Stout, N.: 1989, Publ. Astron. Soc. Pas. 101, 528

Bopp, B.W., Saar, S. H., Ambruster, C., Feldman, P., Dempsey, R., Allen, M., Barden, S.P.: 1989, Astrophys. J. 339, 1059

Borra, E.F., Edwards, G., Mayor, M.: 1984, Astrophys. J. 284, 211

Bruning, D.H., Saar, S.H.: 1990, in Cool Stars, Stellar Systems, and the Sun ed. G. Wallerstein, Provo, ASP press, p. 165

Butler, C.J. et al. : 1987, Astron. Astrophys. 174, 139

Calamai, G., Landi Degl'Innocenti, E., Landi Degl'Innocenti, M.: 1975, Astron. Astrophys. 45, 297

Donati, J.-F., Semel, M., Rees, D., Taylor, K., Robinson, R.: 1990, Astron. Astrophys. 232, L1

Durney, B.R., Robinson, R.D.: 1982, Astrophys. J. 253, 290

Galloway, D.J., Weiss, N.O.: 1981, Astrophys. J. 243, 945

Gilliland, R.L.: 1985, Astrophys. J. 290, 286

Gray, D.F.: 1976, Observation and Analysis of Stellar Photospheres, Wiley, New York

Gray, D.F.: 1984, Astrophys. J. 281, 719

Gray, D.F.: 1988, Lectures on Spectral-line Analysis: $F, G$, and $K$ Stars, Arva

Grossmann-Doerth, U., Schüssler, M., Solanki, S.K.: 1988, Astron. Astrophys. 206, L37

Grossmann-Doerth, U., Solanki, S.K.: 1990, Astron. Astrophys. , in press

Hackman, T., Piskunov, N.E., Poutanen, M., Strassmeier, K.G., Tuominen, I.: 1991, these Proceedings

Hartmann, L., Bopp, B.W., Dussalt, M., Noah, P.V., Klimke, A.: 1981, Astrophys. J. 249,662

Hartmann, L. W., Noyes, R.W.: 1987, Ann. Rev. Astron. Astrophys. 25, 271

Huovelin, J., Saar, S.H.: 1991, these Proceedings

Keller, C.U., Solanki, S.K., Steiner, O., Stenflo, J.O.: 1990, Astron. Astrophys. 233, 583

Kemp, J.C. et al. : 1987, Astrophys. J. Letters 317, 29

Leroy, J.L.: 1962, Ann. d'Astrophys. 25, 127

Landolfi, M., Landi Degl'Innocenti, M., Landi Degl'Innocenti, E.: 1989, Astron. Astrophys. 216, 113

Linsky, J.L.: 1989, Solar Phys. 121, 187

Livingston, W.C.: 1982, Nature 297, 208

MacGregor, K.B., Brenner, M.: 1989, Bull. A. A. S. 21, 1078

Marcy, G.W., Basri, G.S.: 1989, Astrophys. J. 345, 480

Marcy, G.W., Basri, G.S., Valenti, J.A.: 1991, these Proceedings 
Mathys, G., Solanki, S.K.: 1989, Astron. Astrophys. 208, 189

Montesinos, B., Fernández-Villacañas, J.L., Jordan, C.: 1991, these Proceedings

Mullan, D.J.: 1984, Astrophys. J. 279, 746

Radick, R.R., Lockwood, G.W., Skiff, B.A., Baliunas, S.L.: 1988, Bull. A.A.S. 20, 995

Robinson, R.D.: 1980, Astrophys. J. 239, 961

Saar, S.H.: 1988a, Astrophys. J. 324, 441

Saar, S.H.: 1988b, in Hot Thin Plasmas in Astrophysics, ed. R. Pallavicini, Kluwer, Dordrecht, p. 139

Saar, S.H.: 1990a, in IAU Symp. 138, The Solar Photosphere: Structure, Convection, Magnetic Fields, ed. J. O. Stenflo, Kluwer, Dordrecht, p. 427

Saar, S.H.: 1990b, in Mechanisms of Chromospheric and Coronal Heating, in press

Saar, S.H., Linsky, J.L.: 1986, Advances in Space Physics 6, No. 8, 235

Saar, S.H., Bruning, D.H. : 1990, in Cool Stars, Stellar Systems, and the Sun ed. G. Wallerstein, Provo, ASP press, p. 168

Saar, S.H., Neff, J.E.: 1990, in Cool Stars, Stellar Systems, and the Sun ed. G. Wallerstein, PASP Conf. Series, Provo, p. 171

Saar, S.H., Linsky, J.L., Giampapa, M.S.: 1987, in Observational Astrophysics With High Precision Data, eds. L. Delbouille, A. Monfils, U. de Liège, Liège, p. 103

Saar, S.H., Huovelin, J., Giampapa, M.S., Linsky, J.L., Jordan, C.: 1988, in Activity in Cool Star Envelopes, eds. O.Havnes et al., Kluwer, Dordrecht, p. 45

Saar, S.H., Golub, L., Bopp, B.W., Herbst, W., Huovelin, J.: 1990, in Evolution in Astrophysics, ed. E. Rolfe, ESA SP-310, p. 431

Sánchez Almeida, J., García López, J.: 1991, these Proceedings

Schrijver, C.J.: 1990a, Astron. Astrophys. 234, 315

Schrijver, C.J.: 1990b, in Mechanisms of Chromospheric and Coronal Heating, in press

Schrijver, C., Coté, J., Zwaan, C., Saar, S.H.: 1989, Astrophys. J. 337, 964

Semel, M.: 1989, Astron. Astrophys. 225, 456

Simon, T., Herbig, G., Boesgaard, A.M.: 1985, Astrophys. J. 293, 551

Solanki, S.K.: 1986, Astron. Astrophys. 168, 311

Solanki, S.K., Steiner, O.: 1990, Astron. Astrophys. 234, 519

Stenflo, J.0.: 1989, Astron. Astrophys. Rev. 1, 3

Stenflo, J.O., Lindegren, L.: 1977, Astron. Astrophys. 59, 367

Steiner, O., Pizzo, V.: 1989, Astron. Astrophys. 211, 447

Stępień, K.: 1989, Astron. Astrophys. 210, 273

Sun, W.-H., Giampapa, M.S., Worden, S.P.: 1987, Astrophys. J. 312, 930

Toner, C.G., Gray, D.F.: 1988, Astrophys. J. 334, 1008

Toner, C.G., LaBonte, B.: 1990, Astropys. J., in press.

Unno, W.: 1956, Pub. Ast. Soc. Jap. 8, 108.

Valenti, J.A.: 1991, these Proceedings

Vilhu, O.: 1984, Astron. Astrophys. 133, 117

Vogt, S.S.: 1988, in IAU Symp. 132, eds. G. Cayrel, M. Spite, Kluwer, Dordrecht, p. 253.

Walter, F.M. et al.: 1988, Astron. J. 96, 297

Zwaan, C., Cram, L.E.: 1989, in FGK Stars and T Tauri Stars, eds. L.E. Cram, L.V. Kuhi, NASA SP-502, p. 215 